\title{
Movement characteristics and power output of guinea-pig and hamster spermatozoa in relation to activation
}

\author{
D. F. Katz*, R. Yanagimachi and R. D. Dresdner* \\ * Department of Mechanical Engineering, University of California, Berkeley, California 94720, and \\ Department of Anatomy and Reproductive Biology, University of Hawaii School of Medicine, \\ Honolulu, Hawaii 96822 , U.S.A.
}

\begin{abstract}
Summary. Spermatozoa were collected from the cauda epididymidis of golden hamsters and guinea-pigs, and the acrosome reaction was induced in vitro. Movement characteristics of the spermatozoa were assessed with high-speed cinemicrography. Before the initiation of the acrosome reaction (preactivated spermatozoa), sperm movement in both species was characterized by progressive swimming by regular flagellar waves of moderate amplitude and relatively high frequency. After the acrosome reaction (activated spermatozoa), sperm movement in both species was not progressive, and was characterized by whiplash-like flagellar undulations of significantly $(P<0.05)$ higher amplitude and lower frequency. Calculation of the hydrodynamic power output by a new theory indicated that no significant change occurred after activation.
\end{abstract}

\section{Introduction}

Despite the considerable attention paid to the behaviour of mammalian spermatozoa, it is not established whether the spermatozoa manifest the same pattern of flagellar movement from the moment of ejaculation until the time of fertilization. Yanagimachi (1969a, b, 1970b) noted that the pattern of fiagellar movement of hamster spermatozoa incubated in sperm-capacitating media changes after the spermatozoa have been capacitated and undergone the acrosome reaction. Precapacitated spermatozoa with intact acrosomes propagate waves of moderate amplitude along their flagella. In contrast, capacitated and acrosome-reacted spermatozoa display more visually vigorous motility, characterized by whiplash-like waves of significantly greater amplitude. Spermatozoa showing movement of the latter type have been referred to as 'activated' (Yanagimachi, 1970b). Hamster sperm activation in vitro does not appear to be entirely unphysiological, because spermatozoa within the oviducts of naturally mated females display typically activated movement at the site and time of fertilization (Yanagimachi, 1970b).

It is not known at present whether sperm activation is a universal phenomenon. It has been observed in vitro for guinea-pig (Yanagimachi, 1972; Barros, Berrios \& Herrera, 1973; Yanagimachi \& Usui, 1974), dog (Mahi \& Yanagimachi, 1976), and possibly mouse (Iwamatsu \& Chang, 1969) spermatozoa. However, the functional significance of sperm activation in the fertilization process is not clear. The purpose of the present study was to assess sperm motility before and after activation, and to investigate certain hydrodynamic consequences of the two different types of movement. Of particular interest was the rate of hydrodynamic energy expenditure, i.e. the power output, and comparisons of the propulsive thrust and fluid motions induced, for their relationship to sperm activity within or in close proximity to the egg investments.

\section{Materials and Methods}

Preactivated and activated spermatozoa were obtained from four golden hamsters as follows. Spermatozoa were collected from the distal cauda epididymidis, pooled and suspended at a concentration of approximately $10^{7}$ spermatozoa $/ \mathrm{ml}$ in one or two parts Tyrode's solution containing $0.1 \%$ bovine serum albumin (Tyrode-BSA) + one part preheated $\left(56^{\circ} \mathrm{C}, 30 \mathrm{~min}\right)$ rabbit blood serum.

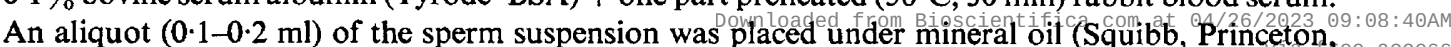


New Jersey) in a watchglass and incubated at $37^{\circ} \mathrm{C}$ under an air atmosphere. Under such conditions, spermatozoa began activation and the acrosome reaction in 3-4 h (Yanagimachi, 1970a). In general, the initiation of activation slightly preceded that of the acrosome reaction. Samples of preactivated and activated spermatozoa were taken after incubation for $<10 \mathrm{~min}$ and $4-5 \mathrm{~h}$ respectively.

Guinea-pig spermatozoa were collected from the distal cauda epididymidis of four animals, pooled and suspended in Tyrode-BSA or a modified Krebs-Ringer solution (BWW medium; see Biggers, Whitten \& Whittingham, 1971) at a concentration of approximately $2-4 \times 10^{6}$ spermatozoa/ $\mathrm{ml}$. An aliquot $(0 \cdot 1-0.4 \mathrm{ml})$ of the sperm suspension was placed under mineral oil in a watchglass and incubated at $37^{\circ} \mathrm{C}$ under an air atmosphere. Preactivated spermatozoa were those incubated for $<2 \mathrm{~h}$. In some cases, spermatozoa were incubated for $2-3 \mathrm{~h}$ in media containing $0 \cdot 2-0 \cdot 4 \%$ bovine pancreatic trypsin (twice crystallized; ICN Pharmaceutical Co., Cleveland, Ohio) to free the spermatozoa from the head-to-head stackings commonly observed among epididymal spermatozoa. Sperm activation and the acrosome reaction were induced by subjecting fresh epididymal spermatozoa to BWW medium containing 0.003\% Hyamine 2389 (Rohm/Haas, Philadelphia, Pa). Under such conditions most spermatozoa display activation and the acrosome reaction within 5-10 min, and thereby attain the ability to fertilize eggs (Yanagimachi, 1975).

Cine films were taken at 100 frames/sec with a Redlake Locam 16-mm high-speed camera. Stroboscopic illumination was correlated to the camera shutter. A timing light generator provided a precise measure of the framing rate. Dry phase-contrast optics were utilized, at magnifications of $\times 200$ for guinea-pig spermatozoa and $\times 160$ for hamster spermatozoa. Slide preparations consisted of approximately $40 \mu \mathrm{l}$ sperm suspension pipetted onto a preheated slide and covered by a preheated $18 \times 18 \mathrm{~mm}$ coverslip (No. $1 \frac{1}{2}$ ) rimmed with vaseline. The resulting preparations were approximately $120 \mu \mathrm{m}$ deep and possessed adequate depth for the spermatozoa to swim freely. The preparations were maintained at $37^{\circ} \mathrm{C}$ throughout the filming procedure by a heated lucite chamber which enclosed the microscope stage. Before filming, each new slide preparation remained on the stage for at least 2 min to achieve thermal equilibrium.

Processed cine films were analysed frame-by-frame on a Vanguard Motion Analyzer. At least 40 freely swimming spermatozoa in each preparation were randomly chosen for measurement. An average flagellar beat frequency was determined by measuring the duration of 10 or more consecutive beats. Representative values of flagellar wavelength and amplitude were also measured for this beat sequence. The former was determined by reference to an approximate axis of symmetry of the beat, which was defined as one-half the maximum transverse displacement from this axis. An effective number of wavelengths was noted. The speed of the sperm head during each beat sequence was determined by measuring the length per unit time of the trajectory of the junction point between the head and the midpiece. Statistical comparisons of mean values of movement characteristics employed Student's $t$ test.

On the basis of the mean values of the preceding movement characteristics mathematical computations were performed of the hydrodynamic power output of representative spermatozoa. The expenditures due to movements of the fiagellum and the head were calculated separately and then summed. For the former, a beat was approximated as sinusoidal, and with prescribed wavelength, amplitude, frequency, and number of wavelengths on a flagellum of known diameter. These parameters served as the input to a computer programme which calculated the power output by means of a new hydrodynamic theory (Dresdner, 1977). This theory is more accurate than previous formulae for power output (Carlson, 1959; Rothschild, 1961), which are only strictly applicable to very small amplitude waves on flagella containing very large numbers of wavelengths. In contrast, our theory is applicable to more realistic beats in which the amplitude is comparable in magnitude to the wavelength, and in which the flagellum contains a small, possibly non-integral number of wavelengths. A summary of the method is given in the Appendix. In the computations for guinea-pig spermatozoa, the flagellum was taken as a cylinder of radius $0.25 \mu \mathrm{m}$ and length $98 \mu \mathrm{m}$; for hamster spermatozoa the radius was taken as $0.4 \mu \mathrm{m}$ and the length as $182 \mu \mathrm{m}$. These values were from measurements of spermatozoa examined by light microscopy.

The power expenditure, $\boldsymbol{P}_{\mathrm{H}}$, due to the movements of the sperm head was calculated from the formula $P_{\mathrm{H}}=6 \pi \eta a \mathrm{~K} V_{\mathrm{H}}{ }^{2}$ (see Happel \& Brenner, 1965). The head is considered geometrically to be 
an oblate spheroid of major radius $a$, the factor $\mathrm{K}$ depending upon $a$ and the minor radius, $b$, and the direction of movement relative to the spheroid axis. The speed of the sperm head is $V_{\mathrm{H}}$ and the viscosity of the suspending medium is $\eta$. The power output $P_{\mathrm{H}}$ is in general not sensitive to the fine details of the geometry of the head, and is generally small in comparison with the power output due to flagellar undulations. The computations for a guinea-pig sperm head used the values $a=4.5 \mu \mathrm{m}$, $b=2 \mu \mathrm{m}$ for spermatozoa with an intact acrosome and $a=3.5 \mu \mathrm{m}, b=0.9 \mu \mathrm{m}$ for spermatozoa without an acrosome; the equivalent values for hamster spermatozoa were $a=4.5 \mu \mathrm{m}, b=2 \mu \mathrm{m}$ (with acrosome) and $a=3.25 \mu \mathrm{m}, b=1.75 \mu \mathrm{m}$ (no acrosome). The viscosity was taken as that of water at $37^{\circ} \mathrm{C}, \eta=7 \times 10^{-4} \mathrm{~Pa} / \mathrm{sec}$.

\section{Results}

The movement characteristics observed and the power outputs calculated are summarized in Table 1 ; the trends were similar for both species. Visually, the shapes of the movement patterns appeared identical to those illustrated previously (Yanagimachi, 1970b). Activated movement was clearly distinguished from preactivated movement by substantially lower values of beat frequency and higher values of beat amplitude, without, however, substantial changes in wavelength. Activated movement was characterized by a beat form containing one, or slightly less, wavelength; preactivated movement characteristically contained approximately $1 \frac{1}{4}$ wavelengths. There was no appreciable difference in power outputs between the two types of movement. In the calculations of total power output (W/ spermatozoon), the expenditure due to the movements of the head $\left(\boldsymbol{P}_{\mathbf{H}}\right)$ made relatively small contributions: for guinea-pig spermatozoa this was $1.92 \times 10^{-15}$ for preactivated movement and $1.29 \times$ $10^{-14}$ for activated movement; for hamster spermatozoa $P_{\mathrm{H}}$ was $3.84 \times 10^{-15}$ for preactivated and $1.52 \times 10^{-14}$ for activated spermatozoa. Typical preactivated sperm head speeds were $V_{\mathbf{H}}=165-222$ $\mu \mathrm{m} / \mathrm{sec}$ for guinea-pig and $V_{\mathrm{H}}=319-471 \mu \mathrm{m} / \mathrm{sec}$ for hamster spermatozoa. The trajectories of the head in preactivated movement were relatively straight. In contrast, activated movement was characterized by large lateral and fore-to aft displacements of the head, with magnitude similar to that of the beat amplitude. The head speed varied during these displacements in the range $V_{\mathrm{H}}=400$ $850 \mu \mathrm{m} / \mathrm{sec}$ for both species, a typical mean value being $600 \mu \mathrm{m} / \mathrm{sec}$.

Table 1. Movement characteristics and total power output for guinea-pig and hamster spermatozoa (mean \pm s.e.m. of 40 spermatozoa for each observation)

\begin{tabular}{|c|c|c|c|c|c|}
\hline Species & Movement type & $\begin{array}{l}\text { Beat frequency } \\
(\mathbf{H z})\end{array}$ & $\begin{array}{l}\text { Amplitude } \\
(\mu \mathrm{m})\end{array}$ & $\begin{array}{l}\text { Wavelength } \\
(\mu \mathrm{m})\end{array}$ & $\begin{array}{l}\text { Total power } \\
\text { output } \\
\text { (W/10 } \\
\text { (14 sperm.) }\end{array}$ \\
\hline Guinea-pig & $\begin{array}{l}\text { Preactivated } \\
\text { Tyrode-BSA* } \\
\text { BWW* }\end{array}$ & $\begin{array}{l}17.2 \pm 0.72 \\
22.7 \pm 0.58\end{array}$ & $7 \cdot 13 \pm 0.12 \dagger$ & $86 \cdot 1 \pm 2.25 \dagger$ & $\begin{array}{l}5.08 \pm 0.23 \\
8.71 \pm 0.41\end{array}$ \\
\hline Hamster & $\begin{array}{l}\text { Activated } \\
\text { Preactivated } \\
\text { Activated }\end{array}$ & $\begin{array}{l}7 \cdot 71 \pm 0 \cdot 19 \ddagger \\
12 \cdot 4 \pm 0 \cdot 41 \\
7 \cdot 11 \pm 0 \cdot 17 \ddagger\end{array}$ & $\begin{array}{c}13.4 \pm 0.47 \ddagger \\
8.6 \pm 0.26 \\
17.0 \pm 0.43 \ddagger\end{array}$ & $\begin{array}{r}85.6 \pm 2.81 \\
111 \pm 2.33 \\
102 \pm 3.06\end{array}$ & $\begin{array}{l}5.19 \pm 0.29 \\
5.81 \pm 0.27 \\
6.57 \pm 0.31\end{array}$ \\
\hline
\end{tabular}

* Media $+0.2 \%$ or $0.4 \%$ trypsin.

$\dagger$ These results from the two media were pooled because there was no significant difference $(P>0.05)$ between the separate means (total of 80 spermatozoa).

$\ddagger$ These values are significantly different from the corresponding values for preactivated spermatozoa, $P<0.05$.

\section{Discussion}

This study represented an initial attempt to quantify the changes in sperm movement associated with the activation process, and to interpret them in the light of current knowledge of sperm physiology. It is clear that sperm activation in vitro causes substantial changes in fiagellar beat shape, in particular

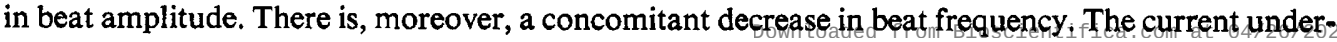
standing of the flagellar contraction mechanism in spermatozoa distinguishes between factors that 
modulate beat frequency and beat shape. Beat frequency is associated with intermediary metabolism (Gibbons \& Gibbons, 1972; Lindemann, 1976; McGrady \& Nelson, 1976), while beat shape is associated with the sperm membrane potential (McGrady \& Nelson, 1972, 1973) and the forces and bending moments acting upon the spermatozoon (Gibbons, 1975; Brokaw, 1975). We have observed typical preactivated and activated movement on headless guinea-pig and hamster spermatozoa, as well as activated movement by spermatozoa with intact acrosomes (Yanagimachi \& Usui, 1974; unpublished observations). Therefore, while the loss of the acrosomal cap does reduce the viscous drag on the head of a guinea-pig or hamster spermatozoon, it seems unlikely that this relatively small change is sufficient to provoke the striking change in beat shape associated with activation, and a basic alteration of the flagellar contraction mechanism is indicated.

On the basis of the movement characteristics measured in our study it is possible to estimate the speed at which the bending contractions are being propagated along the flagellum, i.e. approximately equal to the product of wavelength and beat frequency divided by the mean cosine of the tangent angle of the flagellum relative to its mean axis. This speed was typically $1600 \mu \mathrm{m} / \mathrm{sec}$ for guinea-pig and $1400 \mu \mathrm{m} / \mathrm{sec}$ for hamster spermatozoa, and did not change substantially when activation occurred. Since beat frequency and bend propagation speed are uniquely related only if the beat shape does not change, these two parameters must therefore be regarded as separate measures of the flagellar activation process.

Our calculations suggest that the hydrodynamic power output of a guinea-pig or hamster spermatozoon does not change appreciably due to activation in vitro. While these computations were approximate, their accuracy was compatible with the overall objectives of this preliminary study. At the suspension depth of $120 \mu \mathrm{m}$, the microscope slide and coverglass had a small influence upon the rates of working of those spermatozoa which were in the midplane of the suspension and were filmed (Katz \& Blake, 1975; Katz, Blake \& Paveri Fontana, 1975). The lack of change in power output does not necessarily imply that no shift in metabolic activity occurs with activation. Work on preactivated bull spermatozoa suggested that the hydrodynamic power output constitutes a relatively small fraction (about $11 \%$ ) of the free energy potentially available from ATP hydrolysis (Rothschild, 1961). The efficacy of this parameter as an indicator of the functional status of spermatozoa is therefore uncertain. Further studies of the activation process, involving simultaneous measurement of biochemical as well as biophysical parameters, are obviously needed.

The power output of a spermatozoon increases continuously as the amplitude of the flagellar beat increases. The progressive swimming speed of a free spermatozoon first increases with beat amplitude and then decreases. The optimum beat shape, as regards hydrodynamic propulsive efficiency (Pironneau \& Katz, 1974, 1975), is consequently not unlike that of the preactivated movement measured here as well as that of many other mammalian spermatozoa including those of bulls (Rikmenspoel, 1962), rams (Denehy, 1975), and our own studies in men and monkeys (D. F. Katz \& D. M. Phillips, unpublished observations).

Flagellar undulations of very large amplitude, e.g. activated movement, are not an effective means of directionally propelling a free swimming spermatozoon in a simple fluid. This does not imply that activated movement has no relation to sperm behaviour near the cumulus and penetration of the egg investments. Large-amplitude flagellar undulations provide a maximal thrust to a stationary spermatozoon (Lighthill, 1976), which would be beneficial to such penetration. Moreover, they induce a maximal stirring of the fluid surrounding the spermatozoon (Lighthill, 1976) which would be advantageous in distributing chemicals amongst spermatozoa when they are near the cumulus cells.

Once a spermatozoon has attained the vicinity of an egg in the ampulla of the oviduct, it must make contact with the cumulus oophorus. It is therefore necessary for the spermatozoon to traverse as large a space as possible close to the cumulus. Progressive, unidirectional swimming is not particularly efficient for this purpose; and it would seem more reasonable for a sperm head to sweep out a greater spatial volume per unit time, via large fore-to-aft and lateral displacements. The higher the speed of such undulatory movements, the greater would be the effective volume swept out by the sperm head, and thus the greater the likelihood of contact with the cumulus. 


\section{Appendix}

A summary of the mathematical basis for the computation of the hydrodynamic power output of a swimming spermatozoon

A detailed description of the mathematical methodology is given by Dresdner (1977). The power output $P$ is derived from the following expression:

$$
P(\mathrm{t})=\int_{0}^{\mathrm{L}} \mathrm{f}(\mathrm{s}, \mathrm{t}) \cdot \mathrm{v}(\mathrm{s}, \mathrm{t}) \mathrm{ds}+6 \pi \eta a \mathrm{~K} \mathrm{~V}_{\mathrm{H}}^{2}
$$

Here $f$ is the local vectorial force/unit length of the flagellum acting on the suspending fluid, and $\mathbf{v}$ is the local absolute velocity of the flagellum; $t$ is time and $s$ is a running variable measured along the centre line of the flagellum, which has total length $L$. The second term in (1) represents the contribution to $P$ from the movements of the sperm head, as defined in the text. Computation of $P$ thus requires a knowledge of $f$ and $v$. The latter is comprised of the beat velocity of the spermatozoon plus the resulting velocity of propulsion, $V_{p}$. While $V_{p}$ can in principle be measured experimentally, $f$ must be obtained from the governing hydrodynamic equations for the suspending fluid in the presence of the swimming spermatozoon, and the equations of motion for the sperm body itself. These coupled equations (see Hancock, 1953; Happel \& Brenner, 1965; Dresdner, 1977) can be formally expressed as:

$$
\begin{gathered}
v(s, t)=\int_{0}^{L} \mathbf{f}\left(s^{\prime}, t\right) \cdot G\left(s^{\prime}, s, t\right) d s^{\prime} \\
\int_{0}^{L} \mathbf{f}(s, t) d s+6 \pi \eta a K_{H} V=0 \\
\int_{0}^{L} r(s, t) \wedge f(s, t) d s+r\left(s_{H}, t\right) \wedge 6 \pi \eta a K V_{H}=0
\end{gathered}
$$

Here $\mathbf{G}$ is the tensorial Green's function representing the response of the fluid to local flagellar movements; $r$ is the position vector to the point on the flagellum given by $s$, the position of the head being denoted $s_{H} ; s^{\prime}$ is defined in the same way as $s$. Equation 2 is the fluid equation of motion and Equations 3 and 4 represent the force equilibrium and the moment equilibrium, respectively, for the sperm body. In this formulation, hydrodynamic interactions between the sperm head and flagellum have been neglected, as has the spin torque on the head. The resulting loss of accuracy is sufficiently small to be consistent with the objectives of this study. A more accurate formulation is described by Dresdner (1977). Previous hydrodynamic analyses and computations of power output (Carlson, 1959; Rothschild, 1961) have been based upon an approximate form of Equation 2; Equation 3 was reduced to a single scalar equation, and Equation 4 was neglected altogether.

Our method of solution is to decompose Equation 2 to the form:

$$
v(s, t)=f(s, t) \cdot \int_{s-\delta}^{s+\delta} \mathbf{G}\left(s,{ }^{\prime} s, t\right) d s^{\prime}+\left(\int_{0}^{s-\delta}+\int_{s+\delta}^{L} f\left(s^{\prime}, t\right) \cdot G\left(s^{\prime}, s^{\prime}, t\right) d s^{\prime}\right.
$$

where the interval $(s-\delta, s+\delta)$ is a small region of length $2 \delta$ about the point $s$ within which $f$ is effectively invariant. The governing system of Equations 3-5 is then solved iteratively, the second term on the right hand side of Equation 5 being treated as a higher order effect. Our solutions were performed numerically on a CDC 7600 computer. The flagellum was apportioned into 60 segments per wavelength, and 16 time steps were taken per beat. Spatial integrations along s were performed with a local parabolic scheme. The flagellar beat was approximated as sinusoidal, with given frequency, amplitude wavelength, and number of wavelengths. The head velocity $V_{H}$ was measured directly. The values of power output given in the text and tables were then computed from Equation and time-averaging over one beat cycle. 


\section{References}

Barros, C., Berrios, M. \& Herrera, E. (1973) Capacitation in vitro of guinea pig spermatozoa in a saline solution. J. Reprod. Fert. 34, 547-549.

Biggers, J.D., Whitten. W.K. \& Whittingham, D.G. (1971) The culture of mouse embryos in vitro. In Methods in Mammalian Embryology, pp. 86-116, Table 6-5. Ed. J. C. Daniel, Jr. Freeman, San Francisco.

BroKaw, C.J. (1975) Spermatozoan motility: a biophysical survey. Biol. J. Linn. Soc. 7, Suppl. 1, 423439.

Carlson, F.D. (1959) The motile power of a swimming spermatozoon. In Proc. Ist Natl. Biophys. Conf. pp. 443-449. Eds H. Quastler \& H. J. Morowitz. Yale University Press, New Haven.

DENEHY, M.A. (1975) The propulsion of nonrotating ram and oyster spermatozoa. Biol. Reprod. 13, 17-29.

DRESDNER, R.D. (1977) Theoretical studies on the motion of self-propelled flagella. Ph.D. thesis, University of California, Berkeley.

Gibsons, B.H. \& Gisbons, I.R. (1972) Flagellar movement and adenosine triphosphate activity in sea urchin sperm extracted with Triton X-100. J. Cell Biol. 54, 75-97.

GibBoNs, I.R. (1975) Mechanisms of flagellar motility. In The Functional Anatomy of the Spermatozoon, pp. 127-140. Ed. B. A. Afzelius. Pergamon Press, New York.

Hancock, G.J. (1953) The self-propulsion of microscopic organisms through liquids. Proc. $R$. Soc. Lond. A 217, 96-121.

HAPPEL, J. \& BRENNER, H. (1965) Low Reynolds Number Hydrodynamics. Prentice Hall, Englewood Cliffs, New Jersey.

Iwamatsu, T. \& Chang, M.C. (1969) In vitro fertilization of mouse eggs in the presence or bovine follicular fluid. Nature, Lond. 224, 919.

KATZ, D.F. \& BLAKE, J.R. (1975) Flagellar motions near walls. In Swimming and Flying in Nature, Vol. I., pp. 173-184. Eds T. Y. T. Wu, C. J. Brokaw \& C. Brennen. Plenum Press, New York.

Katz, D.F., Blake, J.R. \& Paveri Fontana, S.L. (1975) On the movement of slender bodies near plane boundaries at low Reynolds number. J. Fluid Mech. 72, 529-540.

LighTHILl, J. (1976) Flagellar hydrodynamics. S.I.A.M. Rev. 18, 161-230.

LindemanN, C.B. (1976) Adenosine 3':5'-cyclic-monophosphate (cAMP) stimulation of demembranated bull sperm models. J. Cell Biol. 70, 238a, Abstr.
Mahi, C.A. \& Yanagimachi, R. (1976) Maturation and sperm penetration of canine ovarian oocytes in vitro. J. exp. Zool. 196, 189-196.

McGrady, A.V. \& Nfison, L. (1972) Cationic influences on sperm biopotentials. Expl Cell Res. 73, 192-196.

MCGRAdY, A.V. \& Nelson, L. (1973) Electrophysiology of bull spermatozoa: correlations with motility. Expl Cell Res. 76, 349-352.

MCGrady, A.V. \& Nelson, L. (1976) Cholinergic effects on bull and chimpanzee sperm motility. Biol. Reprod. 15, 248-253.

Pironneau, O. \& Katz, D.F. (1974) Optical swimming of flagellated microorganisms. J. Fluid Mech. 66, 391-415.

Pironneau, O. \& Katz, D.F. (1975) Optical swimming motions of flagella. In Swimming and Flying in Nature, Vol. I, pp. 161-172. Eds T. Y. T. Wu, C. J. Brokaw \& C. Brennen. Plenum Press, New York.

RiKMENSPOEL, R. (1962) Biophysical approaches to the measurement of sperm motility. In Spermatozoan Motility, pp. 31-54. Ed. D. W. Bishop. Am. Assoc. Adv. Sci., Washington, D.C.

RoTHSCHILD, LORD (1961) Sperm energetics. In The Cell and the Organism, pp. 9-21. Eds J. A. Ramsay \& V. B. Wiggleworth. Cambridge University Press.

YANAGIMACHI, R. (1969a) In-vitro capacitation of hamster spermatozoa by follicular fluid. J. Reprod. Fert. 18, 275-286.

YANAGIMACHI, R. (1969b) In vitro acrosome reaction and capacitation of golden hamster spermatozoa by bovine follicular fluid and its fractions. J. exp. Zool. 170, 269-280.

YANAGIMACHI, R. (1970a) In vitro capacitation of golden hamster spermatozoa by homologous and heterologous blood sera. Biol. Reprod. 3, 147-153.

YANAGIMACHI, R. (1970b) The movement of golden hamster spermatozoa before and after capacitation. J. Reprod. Fert. 23, 193-196.

YaNAGIMACHI, R. (1972) Fertilization of guinea pig eggs in vitro. Anat. Rec. 174, 9-20.

YANAGIMACHI, R. (1975) Acceleration of the acrosome reaction and activation of guinea pig spermatozoa by detergents and other reagents. Biol. Reprod. 13, 519-526.

YANAGIMACHI, R. \& UsuI, N. (1974) Calcium dependence of the acrosome reaction and activation of guinea-pig spermatozoa. Expl Cell Res. 89, 161-174.

Received 12 July 1977 